\title{
TOBACCO DENIIRIFICATION, GEL FOR INTERFERON, CEREAL VECTORS, IMMUNOASSAY SYSTEM
}

TITLE: Thermophilic Denitrification of Tobacco

INVENTORS: Vedpal Malik, Bernard A. Semp, Herman G. Bravo and Daniel M. Teng, all of Richmond, VA, US

ASSIGNEE: Philip Morris Inc., New York, NY, US

FILING \& ISSUING DATA:

PCT Publication No.: WO 83/01180

PCT Publication Date: April 14, 1983

Priority Country: US

Priority Date: October 1, 1981

Many of the carcinogens in tobacco are nitrogen compounds, and reducing the level of these compounds could reduce the hazards of tobacco smoke. The inventors describe a high temperature process where tobacco is exposed to thermophilic organisms under anaerobic conditions. The organisms denitrify the tobacco as a consequence of their own metabolism. Smoke from the treated tobacco contained reduced levels of oxides of nitrogen. The high temperature process may permit development of tobacco products with reduced levels of nitrates and other potentially harmful nitrogen compounds.

TITLE: Method and Composition for Treating a Patient Suffering from an Interferon-Susceptible Disorder

INVENTOR: Kurt F. Berg, Risskov, DK

ASSIGNEE: same as inventor

FILING \& ISSUE DATA:

PCT Publication No.: WO 83/01198

PCT Publication Date: April 14, 1983

Priority Country: DK

Priority Date: October 8, 1981

Interferon treatment of some diseases is particularly effective if the interferon is applied locally because the interferon is active at the cell surface. This patent describes the preparation of a gel composition that can be sterilized, mixed with an interferon solution, and applied locally to treat interferon susceptible disorders. The interferon gel could be used to treat local lesions resulting from Herpes simplex and other viruses. The topical interferon treatment could also treat some kinds of dermatitis, e.g., seborrhea, and precancerous and cancerous tumors.

TITLE: Process for the Genetic Modification of Cereals with Transformation Vectors

INVENTORS: Robert Erwin, Redwood City, CA and Ernest Hubbard, Half Moon Bay, CA, US

ASSIGNEE: International Plant Research Institute, San Carlos, CA, US

FILING \& ISSUING DATA:

PCT Publication No.: WO 83/01176

PCT Publication Date: April 14, 1983

Priority Country: US

Priority Date: October 1, 1981

The patent describes novel transformation vectors for genetic modification of plant cells and, eventually, plant tissue and organs. The vectors are double stranded DNA molecules that contain three essential elements. First, a gene or group of genes identical to plant DNA. The homology between this region and the plant DNA will facilitate insertion of the vector into the plant DNA via recombination. The second element of the vector is a gene coding for a selectable marker such as antibiotic resistance. This element facilitates selection of cells that contain the vector in an active form. The third element is, of course, a gene or group of genes that expresses a desirable trait or codes for a useful product, e.g., a high food value protein. This vector could be used to transfer a wide variety of genes from bacteria, fungi, and other plants to plant cells. It is particularly useful as a gene transfer vehicle for monocotyledonous plants, such as cereals, for which there are no known "natural" vectors.

TITLE: Automated Immunoassay System

INVENTOR: Howard M. Chandler, Kangaroo Ground, VIC, AU

ASSIGNEE: Commonwealth Serum Laboratory, Parkville, VIC, AU

FILING \& ISSUING DATA:

PCT Publication No.: WO 83/0119

PCT Publication Date: March 31, 1983

Priority Country: AU

Priority Date: September 25, 1981

Many diagnostic tests can be performed more accurately and less expensively if they are automated. Heterogeneous immunoassays, which are very sensitive, involve separation of reacted and unreacted material and this step usually requires human manipulation. In the patented system, material to be assayed, e.g., blood or serum, is mechanically added to capillary tubes containing a ligand such as an antigen, an antibody, or a hapten. The tubes are then washed to remove unbound material. In the next step, an assay reagent that specifically recognizes and binds to one or both of the components, e.g., an enzyme conjugate, is added to the tubes. Once the conjugate has bound, the tubes are again washed to remove unbound material. Finally the system automatically adds a solution that is both a substrate for the enzyme and a signal for the presence of the enzyme, e.g., a molecule that changes color when it is cleaved by the bound enzyme. The results are read automatically by an integral component of the system, which is interfaced to a calculator or computer. The system works on a programmed time sequence and can be utilized to assay for many different substances simultaneously.

\section{PATENT ANALYSIS}

by Oskar R. Zaborsky

TITLE: Microbiological Production of Lower Aliphatic Carboxylic Acids

INVENTORS: Marvin P. Bryant, and Barbara R. S. Genthner, both of Urbana, IL, US 